\title{
Prólogo. BioDiseño, Innovación y Transdisciplinariedad
}

Amilton Arruda ${ }^{(1)}$ y Carla Langella ${ }^{(2)}$

\begin{abstract}
Resumen: Este Cuaderno ofrece la oportunidad de revisar la evolución del proyecto bioinspirado a la luz del estado actual del arte, en el que se ubican las aportaciones propuestas, que constituyen los resultados de este camino evolutivo pero también una oportunidad para prefigurar los distintos caminos que conducen al futuro. En la historia de la cultura del diseño, la naturaleza siempre ha ofrecido inspiraciones preciosas, tanto formales como funcionales, capaces de generar nuevas soluciones técnicas y expresivas incluso a problemas de diseño muy complejos.
\end{abstract}

Palabras clave: Diseño - Naturaleza - BioDiseño - Biónica - Innovación - Transdisciplinariedad - Investigación en Diseño - Tecnología Digital - Ciencia

[Resúmenes en inglés y portugués en las páginas 34-35]

(1) Amilton Arruda. Graduation in Industrial Design Product Project by UFPE (1982), Master in Design and Bionics by IED in Milan (1992), Doctorate in Ricerca in Disegno Industriale - Ph.D by University of Milan Politecnico (2002) and postdoctoral in Design and Bionics at IADE European University UNIDCOM Lisbon (2018/2019). Since 1985 professor of the UFPE Design Course. He is currently associate professor IV. He coordinates the Research Group on Biodesign and Industrial Artifacts at UFPE. Organizer together with Edgard Blucher of the [DesignCONTEXTO] series design, culture and technology essays from the following books: (2017) 1. Design and Complexity; (2017) 2. Design and Social Innovation; (2018) 3. Design, Artifacts and Sustainable Systems; (2019) 4. Narratives and Languages in the creative process in Modeling and Prototyping (in press). Always with Edgar Blucher he organizes the series [designNATUREZA] essays on design, bionics and biomimetics with the following book: (2018) Methods and Processes in Bionics and Biomimetics: the technological revolution by nature. With the publisher Insigh in (2019) we launched the following title: Topics in Design: Biomimetics, Sustainability and New Materials; and recently with Blucher (2020) the Book: Design and Bionics. Carmelo Di Bartolo and Centro Ricerche IED: esperienze memorabili da 30 protagonisti.

(2) Carla Langella. Architect, Associate Professor of Industrial Design at the Department of Architecture and Industrial Design, University of Campania "Luigi Vanvitelli", where she teaches Bio-innovation Design and Design for Scientific Visualization in the master's 
degree Course Design for Innovation and Industrial Design Laboratory 3 in the Threeyear Degree Course of Design and Communication. In the field of experimental design research, she investigates the opportunities to build hybrid paths that involve the most advanced scientific contributions in the design project to bring contemporary science closer to people's lives. Since 2006 she founded and coordinates the Hybrid Design Lab (www. hybriddesignlab.org), the design laboratory dedicated to the mutual relations between design and science with particular attention to the experimentation of biomimicry in design and the integration of designers in the development processes of new materials to which the specific Designer in lab project is dedicated.

\section{El potencial de compartir}

En el campo de la investigación del diseño, la oportunidad de trabajar en equipo es siempre una oportunidad preciosa porque permite intercambiar visiones, ideas y valores, explorando territorios siempre insólitos y estimulantes, haciendo de la investigación también una experiencia agradablemente colaborativa. Es aún más emocionante activar la colaboración entre diferentes grupos de investigación, pertenecientes a contextos geográficos y culturales distantes, que tienen objetivos de investigación similares pero enfoques y experiencias diferentes. $Y$ esto es lo que nos sucedió a través de la colaboración entre nuestros grupos de investigación-BiodesignLab de la Universidad de Pernambuco e Hybrid Design Lab de la Universidad de Campania "Luigi Vanvitelli"- que involucró a nuestros jóvenes colaboradores pero también a nuestras redes de empresas, museos e investigación. Centros como D.RE.AM Design and Research in Advance Manufacturing y FabLab de Città della Scienza de Napoli, el laboratorio de fabricación digital LOUCo, FABLab, del programa AIRES y otras actividades en Porto Digital. Una colaboración que comenzó hace solo tres años, gracias a nuestro maestro común Carmelo Di Bartolo que nos puso en contacto, pero que ha sido muy intensa y fructífera desde el principio y ya ha obtenido muchos resultados como publicaciones (incluido el libro Biónica y Diseño, impreso en Portugal y publicado en 2020 por Edgard Blucher en su plataforma OpenAccess), actividades de investigación, cooperación en tesis doctorales (Valentina Perricone y Antonio Roberto), actividades docentes conjuntas y consultas, con muchas hasta reuniones digitales pero también físicas como laboratorio recíproco visitas, conferencias y presentaciones de libros, hasta llegar a esta publicación.

Estamos muy contentos con la oportunidad que se nos ha brindado de ser editores de este texto titulado: Diseño, Innovación y Transdisciplinariedad. Relaciones del Diseño con la Naturaleza, la Biología y la Tecnología dentro de los Cuadernos del Centro de Estudios en Diseño y Comunicación de la Facultad de Diseño y Comunicación de la Universidad de Palermo, porque nos ofrece la oportunidad de mover los hilos, a la luz de nuestro enfoque ahora mutuo, del estado actual del arte en el diseño inspirado en la naturaleza, de las diferencias de enfoque entre contextos de investigación y generaciones de investigadores. Una oportunidad que nos ve colaborando con un socio tecnológico de gran visibilidad 
internacional como es UP Universidad de Palermo, con quien nuestra red de relaciones colaborativas se extiende y se expresa a través de este rico texto.

Del texto han surgido cuatro macrotemas

- Aspectos transdisciplinares y pedagógicos

- Aspectos estratégicos y de innovación

- Aspectos de diseño y naturaleza

- Aspectos de diseño y tecnología

En el apartado Aspectos estratégicos y de innovación los autores contribuyen a una sistematización de los métodos de enseñanza utilizados en el campo del diseño inspirados en la naturaleza y a una clarificación de las distinciones y solapamientos semánticos entre los conceptos de multidisciplinariedad, transdisciplinariedad e interdisciplinariedad. De esto surge un cuadro muy interesante que resalta la importancia de la integración entre el componente teórico-especulativo y el práctico que caracteriza en particular al diseño bioinspirado.

En la parte Aspectos de diseño y naturaleza surgen diferentes orientaciones metodológicas para la innovación del diseño biónico, también a la luz de las nuevas oportunidades que ofrece la evolución de materiales y tecnologías y la aparición de nuevas necesidades de sostenibilidad.

Los aportes del apartado Aspectos de diseño y tecnología ofrecen una visión muy amplia de los sectores de aplicación y sectores productivos en los que el diseño bioinspirado puede aportar ventajas y avances sustanciales como el sector del packaging, la movilidad, las fachadas de edificios, destacando la importancia del contribución de las tecnologías digitales a la aplicación de estos métodos.

\section{La evolución del enfoque bioinspirado del proyecto}

Este Cuaderno ofrece la oportunidad de revisar la evolución del proyecto bioinspirado a la luz del estado actual del arte, en el que se ubican las aportaciones propuestas, que constituyen los resultados de este camino evolutivo pero también una oportunidad para anticipar los distintos caminos que conducen al futuro. En la historia de la cultura del diseño, la naturaleza siempre ha ofrecido inspiraciones preciosas, tanto formales como funcionales, capaces de generar nuevas soluciones técnicas y expresivas incluso a problemas de diseño muy complejos. Todo el mundo conoce ejemplos de innovaciones inspiradas en la naturaleza como las máquinas voladoras de Leonardo Da Vinci (1476) inspiradas en pájaros, el Crystal Palace diseñado por Joseph Paxton (1850) inspirado en una hoja, Velcro inspirado en flores de bardana (1955). En los diversos contextos y épocas, la bioinspiración ha surgido cíclicamente asumiendo distintas connotaciones (La Rocca, 1997), desde las esencialmente formales a las más funcionalistas y estructurales, hasta las evocadoras que emplean la naturaleza con intenciones filosóficas y especulativas. El enfoque que hoy se define como biomimetismo, biónico, biomimético, no es -de hecho-una cosa nueva. 
Sin embargo, en comparación con el pasado, este Cuaderno que proponemos surge de la necesidad de comprender cómo hoy en día un enfoque basado e inspirado en la naturaleza es aún capaz de generar resultados originales e innovadores además de encontrar sutiles diferencias entre diversas investigaciones pasadas y las actuales. Este texto recoge testimonios de algunos de los protagonistas más activos en este campo a nivel internacional que nos permiten construir un escenario actualizado y representativo de lo que es hoy el diseño biomimético. De los capítulos que siguen se desprende que, especialmente los autores más jóvenes, han entendido bien que el enfoque biónico requiere una transdisciplinariedad real, concreta y no teórica, basada en la intersección de habilidades. Una intersección que requiere mucho compromiso y nuevas herramientas críticas y culturales para la figura del diseñador que incluyen, no sólo la capacidad de comprender conceptos científicos sino de saber aplicarlos de forma consciente y de forma rigurosamente científica. Los diseñadores inspirados en la naturaleza de hoy colaboran directamente con biólogos e ingenieros para desarrollar principios y diseños compartidos e híbridos.

De las contribuciones propuestas en el Cuaderno, se desprende también la evolución de las tecnologías digitales, tanto del lado de los instrumentos de escaneo y microscopía utilizados por los biólogos, como del lado de las tecnologías de modelado e impresión 3D utilizadas por los diseñadores, las que han revolucionado el diseño inspirado por biología. Lo digital se ha convertido en un terreno de convergencia entre diseño y ciencia, una oportunidad para discutir archivos para compartir con el fin de realizar simulaciones, modelado e interpretaciones compartidas. Los microscopios SEM y los instrumentos de tomografía, por ejemplo, permiten comprender las estructuras y los fenómenos biológicos en sus más mínimos detalles, cosa que en el pasado no eran observables, debido a los límites de aumento de los instrumentos de microscopía. Por lo tanto, se hace posible analizar y reproducir los procesos generativos, químicos, físicos y moleculares que son la base de esos mecanismos que han fascinado a muchos diseñadores biónicos del pasado, pero que lamentablemente no fueron capaces de explicar o reproducir.

Hoy, a la luz del conocimiento cada vez más profundo de los principios que regulan la complejidad de los sistemas biológicos, el límite de los enfoques funcionalistas, por ejemplo la biotecnología clásica, es evidente al interpretar la naturaleza como un modelo fundamentalmente estático de rigor, perfección y eficiencia absoluta. El proyecto contemporáneo, que pretende trasladar la compleja inteligencia de la naturaleza, también se refiere a principios de adaptación menos exactos, en ocasiones aparentemente ineficaces como la redundancia, que resulta fundamental para asegurar la resiliencia de los sistemas biológicos. En los proyectos descriptos en las distintas contribuciones, los diseñadores observan la naturaleza ya no desde la distancia sino desde un punto de vista cercano -como biólogos- para apreciar los detalles y cualidades más complejos como la autoorganización, la redundancia, la autoadaptación, la autodeterminación, montaje y multifuncionalidad entre otros. Estos principios constituyen las nuevas referencias para un proyecto bioinspirado que debe dar respuesta a necesidades y limitaciones cada vez más complejas. El nivel de profundidad y precisión del conocimiento actual permite a los diseñadores contemporáneos concebir productos y artefactos que reproducen algunos de los personajes más complejos escondidos en el mundo natural. 
Paralelamente a la evolución de las tecnologías de escaneo y microscopía en las últimas décadas, también se ha realizado una profunda revisión de los procesos productivos que incluye la digitalización de maquinaria, innovación de materiales, nanotecnologías, innovación en química, el desarrollo de procesos biosintéticos que evoluciona en la dirección de una nueva forma de "industria bio-digital" (Estévez, Navarro, 2017). De los testimonios recogidos en este texto surge la conciencia de que la evolución del escenario de los productos industriales procede, siguiendo la complejización de tecnologías, materiales y estilos de vida, en la dirección de artefactos cada vez más compuestos, multifuncionales, interactivos, reactivos y cambiantes, cada vez más similares en características y funcionalidad a los sistemas biológicos. Las herramientas, estrategias y métodos de diseño y producción adecuados a esta complejidad deben, por tanto, referirse al mundo biológico y a los conocimientos más avanzados relacionados con él para poder sustentar esta orientación evolutiva (Langella, 2007).

En el Cuaderno, todos los autores investigadores convergen el principio según el cual el diseño que hace referencia a la biología se basa en analogías entre problemas de diseño y problemas de la vida, y en la abstracción que nos permite traducir caracteres biológicos en caracteres artificiales o híbridos. Los enfoques de diseño descriptos se basan en la transferencia de conocimientos, principios, lógica, funcionalidad y estructuras extraídas de la biología, a los procesos de innovación de productos y servicios, con el fin de hacerlos más bellos, funcionales, sostenibles, adaptables o adherentes a la nuevas y complejas formas de vivir del mundo contemporáneo. Para definir estos enfoques se utilizan múltiples términos como: diseño biónico, biomimetismo, biomimética y diseño bioinspirado (Nagel, 2014).

Dentro del campo de la literatura especializada internacional, los textos que han arrojado más luz sobre las diversas terminologías, informando también sobre su génesis y desarrollo, emergen autores como Gruber, P. (2011) Biomimética en arquitectura. Springer Verlag Wien, pp.9-49 y Nachtigall, W. y Wisser, A. (2014) Biónica con ejemplos. Heidelberg: Springer. Según Werner Nachtigall, el término Bionik proviene de la palabra inglesa "bionics", que fue utilizada por primera vez por el comandante de la Fuerza Aérea de EE. UU. J. E. Steele en una conferencia titulada "Simposio de biónica: prototipos vivos: la clave de la nueva tecnología" en 1960, probablemente combinando las palabras "biología" y "técnica" o "electrónica". La norma ISO 18458. Biomimética - Terminología, conceptos y metodología propone una clasificación de terminologías que intenta distinguir y codificar los términos utilizados para indicar este enfoque de diseño en forma de glosario, a través de definiciones muy simples y esenciales.

- Bioinspiración: enfoque creativo basado en la observación de sistemas biológicos.

- Biomimetismo: filosofía y enfoques de diseño interdisciplinario que toman la naturaleza como modelo para enfrentar los desafíos del desarrollo sustentable (social, ambiental y económico).

- Biomimética: cooperación interdisciplinaria de biología y tecnología u otros campos de innovación con el objetivo de resolver problemas prácticos a través del análisis de funciones de sistemas biológicos, su abstracción en modelos y la transferencia y aplicación de estos modelos a la solución. 
- Biónica: disciplina técnica que busca replicar, incrementar o reemplazar funciones biológicas por sus equivalentes electrónicos y / o mecánicos.

Las definiciones proporcionadas por ISO intentan resaltar algunos significados específicos tomados por el enfoque de diseño que se inspira en la biología en relación con los contextos históricos y culturales en los que estas denominaciones nacieron o se desarrollaron. El término biomimetismo, por ejemplo, está fuertemente influenciado por el trabajo de la ecologista Janine Benyus (1997), centrado en la sostenibilidad ambiental, mientras que el carácter mecanicista de la biónica sustantiva se refiere al escenario histórico y tecnológico que se desarrolló alrededor de la cibernética el fines de 1950. La intención de proporcionar descripciones concisas y bien delimitadas en un paisaje tan complejo y articulado ha llevado a la ISO a simplificar demasiado las definiciones, lo que ha hecho que las definiciones no sean muy consistentes con esta complejidad y la forma en que se utilizan estos términos. Sin embargo, de una investigación exhaustiva de la literatura temática internacional de los últimos veinte años se desprende que la biónica, la biotecnología, la biomimética, el biomimetismo y el diseño bioinspirado se utilizan generalmente de manera casi equivalente y que pueden considerarse sinónimos. La elección de utilizar una denominación en lugar de otra deriva más que del enfoque aplicado por el contexto geográfico y cultural de origen de los autores. En los países de habla alemana y de habla hispana, por ejemplo, el término diseño biónico se prefiere generalmente, en Inglaterra el término biomimética, en los Estados Unidos biomimetismo. En Italia, la definición biomimética se utiliza con mayor frecuencia. Varios autores, entre ellos Olga Speck, por otro lado, creen que el diseño bioinspirado es la denominación más amplia y adecuada para comprender los múltiples matices de este enfoque de diseño, incluidos los más técnicos y de ingeniería (Speck et al., 2017) en contraste con la norma ISO que la relega al significado de "enfoque creativo".

Más allá de las discusiones sobre terminología, lo que surge del variado marco internacional y la presencia de una norma ISO es la necesidad de comparar y sistematizar las metodologías y puntos de vista de los diseñadores y grupos de investigación que han adquirido experiencia en estos conceptos en diferentes lugares del mundo. Los aportes propuestos se enmarcan dentro de los dos macroenfoques convencionalmente reconocidos por la literatura internacional especializada en bioinspiración (Cohen, Reich, 2016):

- El enfoque bottom up en el que el proyecto parte de una intuición nacida de la observación de un principio o un carácter encontrado en la naturaleza para tomarlo prestado; $\mathrm{o}$, como suele ser el caso, el enfoque de arriba hacia abajo que busca una solución de diseño en biología para un problema de diseño específico desde una perspectiva de resolución de problemas (Speck, Speck, Beheshti, McIntosh, 2008). La contribución de Perricone et al. y de Roberto et al. por ejemplo, parten de la identificación de características biológicas, principalmente biomecánicas o morfológico-funcionales de los equinoides y algunos frutos para luego elegir el diseño y áreas tecnológicas más adecuadas para su transferencia. En la literatura, el enfoque de abajo hacia arriba a se ha definido de diferentes maneras: basado en soluciones (Helms, Vattam, Goel, Yen, Weissburg, 2008; Gebeshuber, Drack, 2008; Badarnah, Kadri, 2015), basado en problemas (Helms, Vattam, Goel, Yen, Weiss- 
burg, 2008), biología push (ISO TC, 2015), biomimética por inducción y biología al diseño (Baumeister, Tocke, Dwyer, Ritter, Benyus, 2014).

- El enfoque opuesto, definido de arriba hacia abajo, también se define como impulsado por problemas (Fayemi, 2017), basado en problemas (Helms, Vattam, Goel, Yen, Weissburg, 2008; Gebeshuber, Drack, 2008; Badarnah, Kadri, 2015), desafío a la biología (Baumeinster, Tocke, Dwyer, Ritter, Benyus, 2014) tecnología pull (ISO TC, 2015) y biomimética por analogía (Gebeshuber, Drack, 2008). El segundo enfoque puede resultar una valiosa herramienta estratégica y de diseño para las empresas manufactureras porque permite reducir en gran medida el tiempo de innovación de productos y procesos y llegar a soluciones originales capaces de distinguirse de la homologación de la competencia.

Muchos de los aportes se refieren a la posibilidad de utilizar la naturaleza como referente para un proyecto más sostenible. La naturaleza es la mejor fuente de inspiración para diseñar artefactos ambientalmente sostenibles compatibles con la compleja dinámica ambiental actual (Alexandridis, 2016). El proyecto de diseño que colabora con la biología puede ofrecer un valioso aporte a la evolución de la cultura del diseño sostenible, de las estrategias de marketing eco-orientadas y de la conciencia ambiental con nuevas y originales herramientas conceptuales, inspiradas en la naturaleza y su resiliencia (Edwards, 2010). La conciencia cada vez más generalizada de los problemas climáticos y ambientales y el impacto del estilo de vida en la salud y el bienestar, lleva a las personas a elegir lo que perciben más natural y similar a su raíz biológica y a preferir productos que implementen un factor biológico, ya sean materias primas o inspiración de diseño. En un mercado cada vez más inclinado a elegir productos de bajo impacto, la bioinspiración es un eficaz vehículo estratégico para caracterizar, identificar y promover productos eco-sostenibles y promover acciones de innovación eco-orientadas. La posibilidad de difundir, a través de productos destinados al mercado, por tanto para entrar en la vida de las personas, nuevos conocimientos y sensibilidad sobre el medio ambiente, la salud de los hombres y la equidad social, ofrece nuevas y concretas perspectivas para aumentar la conciencia y mejorar la calidad de vida (Bosworth, Macer, Sangaroonthong, Waller, 2011). Por estos motivos, las empresas y organizaciones comerciales son ahora conscientes del gran potencial competitivo de la bioinspiración en términos de atractivo, valor percibido y marketing, a partir de estudios sobre el emprendimiento bioorientado definido como bioemprendedor (Ulhøi, 2015).

Finalmente, desde un punto de vista comunicativo y de sensibilización, al elegir un sistema particular como modelo natural, el diseño automáticamente centra la atención en él, en sus personajes y en sus especificidades. No sólo la atención de uno, sino también la de los usuarios, clientes, productores y, si el proyecto se comunica a los medios de comunicación y se difunde, a la opinión pública.

El diseño inspirado en la naturaleza puede así elegir especies raras, en peligro de extinción o autóctonas de contextos geográficos particulares, para concientizar a un objetivo específico o comunidad sobre la delicada dinámica del ecosistema y resaltar sus características de biodiversidad e identidad para luego transferirlas al proyecto de artefacto innovador. Se pueden destacar fenómenos como, por ejemplo, los efectos del cambio climático para 
sensibilizar a la sociedad e instituciones y ayudar en las políticas de protección de los ambientes y especies protegidos. La elección de los organismos de referencia para ser utilizados como modelo de la naturaleza, por lo tanto, es estratégico (tanto en términos de efectividad de transferencia en la propuesta de soluciones de diseño adecuadas a las necesidades planteadas por el mercado y la sociedad, como en la posibilidad de atraer la atención de la opinión pública y política sobre situaciones ambientales críticas que necesitan atención y apoyo). Los investigadores que se ocupan de temas similares pueden encontrar en el diseño bioinspirado un canal rentable para difundir y dar a conocer los resultados de su investigación de acuerdo con un enfoque de Biología hacia el Diseño, que ve a la biología proponiendo modelos de la naturaleza específicos para diseñar y traducirlos en innovación.

\section{Los principios del proyecto biónico}

Este Cuaderno es también una oportunidad para presentar el proyecto Design Future Lab a la comunidad científica del diseño biónico, que nace de una idea de Carmelo Di Bartolo con nuestra colaboración, con el objetivo de configurar, a través de las herramientas del diseño biónico, un futuro constituído a partir de productos y servicios de alto contenido científico y tecnológico pero, basados en los valores humanísticos de calidad relacional, sinestesia, ligereza y sostenibilidad. Como parte del Design Future Lab, se decidió desarrollar una especie de decálogo de diseño biónico a partir del propuesto en el texto Design and Science (Langella, 2019) y teniendo en cuenta el propuesto por Nachtigall. Estos principios se proponen como una especie de cuadrícula de referencia, basada en la experiencia de investigación y diseño de los proponentes. Un decálogo que se propone como un contenido abierto, susceptible de discusiones y cambios, un estímulo a la reflexión más que una guía absoluta.

Principio 1 - Referirse a los datos científicos de las biociencias El proyecto biónico debe hacer referencia a fuentes científicas fiables reconocidas por la comunidad científica internacional. Por lo tanto, los diseñadores deben poder interpretar la literatura científica especializada y consultar a los científicos interactuando con ellos.

\section{Principio 2 - Inspirarse en la naturaleza en lugar de imitarla}

El proyecto biónico debe ir más allá de la imitación formal de la naturaleza y debe hacer referencia a las lógicas, principios, procesos y estructuras observadas por la biología para traducirlos en nuevos artefactos, a veces incluso con un aspecto completamente diferente a sus modelos de referencia.

\section{Principio 3 - Diseño con enfoque multidisciplinar}

El diseño biónico debe abordarse con un enfoque multidisciplinar en el que, junto a las habilidades de diseño, confluyan los conocimientos y la experiencia de otros campos disciplinares identificados según el campo de aplicación y los modelos naturales elegidos. 
Principio 4 - Producir modelos de la naturaleza en clave metaproyectiva

El proyecto biónico se basa en la construcción de modelos de la naturaleza, los cuales consisten en esquemas gráficos, mapas conceptuales y modelos digitales y/o físicos que modelan los procesos y estructuras observados en el mundo biológico a través de las herramientas de representación e interpretación propias de la cultura del diseño. Estos modelos deben ser elaborados por los diseñadores en forma de metaproyecto con una actitud crítica y prefigurativa en relación al problema de diseño específico que se enfrenta, enfatizando los aspectos transferibles.

\section{Principio 5 - Diseño para establecer analogías y homologías}

El diseño biónico se basa en la construcción de analogías (relación de semejanza, o igualdad, entre dos o más cosas que tienen, en su constitución, en su comportamiento, en sus procesos, algún rasgo común) y homologías (conformidad o equivalencia entre varias partes), términos, elementos, muchas veces con un origen común entre los "problemas de la vida" (reproducirse, sobrevivir, crecer, alimentarse, defenderse, etc.) que enfrenta la biología y los "problemas del proyecto" (reproducibilidad, durabilidad, resistencia mecánica, flexibilidad para diferentes usos, ergonomía, etc.).

Principio 6 - Diseñar para la sostenibilidad ambiental, social y económica

El diseño biónico debe proponer soluciones de diseño sostenibles en términos ambientales, sociales y económicos. Ninguna referencia es más confiable que la biología para indicar las mejores formas de producir de acuerdo con la delicada dinámica del ecosistema. La cultura del proyecto debe mirar la lógica con la que la naturaleza maneja la circularidad de los ciclos de vida; la capacidad de construir relaciones sinérgicas, coevolutivas y colaborativas entre sistemas naturales; la tendencia a la autoorganización; multifuncionalidad y optimización en el consumo de materiales y energía. Esta lógica en biónica se convierten en el referente de diseño para proponer procesos de producción, productos ecológicos y estrategias para la recuperación y uso eficiente de los recursos.

\section{Principio 7 - Contribuir a la evolución de la disciplina}

No existe una metodología única reconocida para diseñar artefactos con referencia a la naturaleza. Existen múltiples métodos, enfoques y puntos de vista, así como, en diferentes lugares del mundo, se utilizan diferentes definiciones para identificar el proyecto inspirado en la biología, incluyendo biomimética, biomimetismo, biomimesis o diseño bioinspirado. Como en la naturaleza, toda experiencia exitosa es una evolución que contribuye al crecimiento y difusión de la disciplina.

Principio 8 - Transferencia de las cualidades de resiliencia, flexibilidad y adaptabilidad de la naturaleza

El diseño biónico transfiere a los artefactos la adaptabilidad y flexibilidad de la naturaleza que es capaz de adaptarse de manera resiliente a las cambiantes condiciones internas $y$ externas. Estas cualidades hacen que el enfoque del diseño bioinspirado sea adaptable con respecto al surgimiento de nuevas demandas de innovación y las necesidades de la sociedad contemporánea. 


\title{
Principio 9 - Observar los detalles de la naturaleza
}

Hoy, en comparación con el pasado, la tecnología ofrece herramientas de investigación con resoluciones cada vez mayores que se esfuerzan por observar la naturaleza hasta la escala nanométrica, revelando sus detalles más intimos. A partir de tales detalles, los científicos pueden deducir nuevos conocimientos sobre los procesos y principios detrás de la belleza de las estructuras biológicas. La belleza de la naturaleza está relacionada con su "inteligencia" y es una belleza formada por nervaduras, capas superpuestas, estructuras jerárquicas, porosidad, detalles complejos en todas las escalas dimensionales. Cada una de estas "soluciones detalladas" a menudo realiza más de una función biológica.

\section{Principio 10 - Innovación bienvenida}

El proyecto inspirado en la biología favorece el uso de nuevos materiales, nuevos procesos de producción y nuevas tecnologías porque se adaptan mejor a traducir las complejas cualidades de la naturaleza, concretándolas en nuevos artefactos.

No terminaríamos esta presentación sin manifestar nuestro profundo agradecimiento a la Universidad de Palermo en la persona de la Profesora Daniela V. Di Bella, por comprender la prioridad de valorar esta área del conocimiento, y también a todos nuestros colegas e investigadores de diversas partes del mundo, quienes aceptaron el desafío de enviar parte de sus producciones en el contexto indicado.

Creemos en el legado que estamos proponiendo a las nuevas generaciones de futuros diseñadores y esperamos que sean más conscientes y generosos con la naturaleza.

Vizela e Aversa, 03 mayo 2021

\section{Prof. Ph.D. Amilton Arruda}

Dpto. de Diseño Industrial

Universidad Federal de Pernambuco, Recife, PE, Brasil,

\section{Prof. Doctora Carla Langella}

Dpto. de Arquitectura y Diseño Industrial

Universidad de Campania Luigi Vanvitelli, Aversa, Italia

\begin{abstract}
This Cuaderno offers the opportunity to review the evolution of the bio-inspired project in the light of the current state of the art, in which the proposed contributions are located, which constitute the results of this evolutionary path but also an opportunity to prefigure the different paths that lead to future. In the history of design culture, nature has always offered precious inspirations, both formal and functional, capable of generating new technical and expressive solutions even to very complex design problems.
\end{abstract}


Keywords: Design - Nature - BioDesign - Bionics - Innovation - Transdisciplinarity - Design Research - Digital Technology - Science

Resumo: Este Caderno oferece a oportunidade de rever a evolução do projeto bioinspirado à luz do estado da arte atual, no qual se situam as contribuições propostas, que constituem os resultados deste caminho evolutivo, mas também uma oportunidade de prefigurar o diferente caminhos que levam ao futuro. Na história da cultura do design, a natureza sempre ofereceu inspirações preciosas, tanto formais quanto funcionais, capazes de gerar novas soluções técnicas e expressivas até mesmo para problemas de design muito complexos.

Palavras chave: Design - Natureza - BioDesign - Biônica - Inovação - Transdisciplinaridade - Pesquisa em Design - Tecnologia Digital - Ciência

[Las traducciones de los abstracts fueron supervisadas por el autor de cada artículo] 